\title{
Free space quantum key distribution based on turbulence bubble model
} Xue Yang ${ }^{1 *}$, Ma Li-Hua ${ }^{1}$,Shi Lei ${ }^{1}$, Wei Jia-Hua ${ }^{1}$, Zhu Qiu-Li ${ }^{1}$, Zhu Yu ${ }^{1}$

\author{
1 Information and Navigation College, Air Force Engineering University, Shanxi Xi'An, 710077
}

*Email: relnix@sina.cn

\begin{abstract}
Key words: free space quantum key distribution; turbulence bubble model; polarization state; quantum bit error rate

Abstract: Aiming at the transmission of polarization encoded single photons in turbulent atmosphere, the random turbulence is modeled as atmospheric bubbles with various refractive indexes so as to conceive the propagation process of single photon using geometric optics. The fluctuation of photon transmission ratio $\mathrm{k}$ has been quantitatively calculated which is revealed as the relationship between transmission ratio and incident angle or turbulence scale. Based on that, the quantum bit error rate(QBER) and polarization fidelity of single photons are analyzed respectively which are both caused by the misalignment of polarization state after continuous refractions. Then the three dimensional relationship of $\mathrm{k}$ and polarization error rate $\mathrm{Q}$ are simulated with random incident angle or turbulence scale.
\end{abstract}

\section{Introduction}

With the help of free space quantum key distribution (QKD) two distant parties can share secure random keys even between air and ground, which is regarded as a vital approach to establish secure aeronautical communication link and global confidential network. It has become one of the most promising and practical technology in quantum information field since proposed. So far the study of free space QKD is mainly about its feasibility analysis and security proof [1]. The Villoresi group from Padova realized transmission of single photons between satellite and ground station for the first time by using corner-cube retroflector which promoted the development of free space quantum communication [2]. In 2010 a 16km quantum teleportation has been reported by Pan's group from University of Science and Technology of China which broke the distance record of free space quantum communication [3]. The world's first airborne QKD experiment was implemented by Germany researchers in 2013 in aeronautical environment [4]. Nie analyzed the influence on free space QKD of sandstorm and haze as well as rainfall in detail [5,6], the relationship between quantum channel entanglement and turbulence intensity is established in [7] which serves as theoretical reference for the study of aeronautical quantum communication performance. Since polarization encoding is generally used in free space QKD [8], the signal photon will be influenced by many factors when transmitting in atmospheric channel where the refraction of dielectric interface contributes greatly to the polarization state change and finally induces quantum bit error rate (QBER) [9]. Therefore it is meaningful to figure out the polarization state change of photons after turbulence refraction and the consequent fluctuation of QBER.

\section{The turbulence bubble model}

As polarization encoded single photon propagates in the turbulent atmosphere [10], it is inevitable to be influenced by the refractions of turbulence which will impede the transmission and fidelity of polarization state. The light will be curved due to the inconsistent refractive indexes inside and outside the turbulence [11,12]. And from the microscopic perspective, the $s$ component and $p$ component of polarization state will be changed. In order to simplify the situation the turbulence can be abstracted as air bubbles with random size and refractive indexes. The refractive index inside the bubble will decrease with the enlargement of turbulence scale. Suppose that the refractive index of air is $n_{1}$ and the one inside the bubble is $n_{m}$. And according to [13] there is a relationship between $n_{m}$ and bubble radius $r$ which can be expressed as equation (1) and Figure.1 shows the diagram of photon path through continuous refractions. 


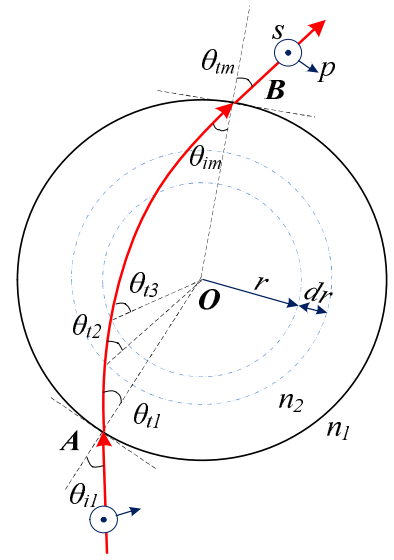

Figure 1. Diagram of single photon path through continuous refractions

$$
n_{m}=n_{1} \sqrt{1-b\left(\frac{r}{a}\right)^{2}}
$$

Where $\mathrm{a}$ and $\mathrm{b}$ are both constant related to temperature and pressure of the turbulence. Suppose that the photon enters at $A$ on the surface of bubble with incident angle of $\theta_{i 1}$ and then emerges out from $B$ point. The refractive angle of the first refraction is $\theta_{t 1}$ which also equals the incident angle of the next refraction. The left incident angles and refractive angles are $\theta_{i 1, i 2 \ldots i m}$ and $\theta_{t 1, t 2 \ldots t m}$ respectively. And $O$ is the center of this bubble. If the wave vector of incident light $i$ is $\stackrel{v}{E}$ and its two components of incident light can be written as follow according to representation of polarized light and Fresnel principle [14], when the refractive index of turbulence is constant there will be:

$$
\begin{aligned}
& \frac{\stackrel{v^{t 1}}{E^{t 1}}}{\underbrace{i 1}_{s}}=\frac{2 \cos \theta_{i 1} \sin \theta_{t 1}}{\sin \left(\theta_{i 1}+\theta_{t 1}\right)} \\
& \frac{\stackrel{\mathrm{E}}{t 1}^{t 1}}{\vec{\vee}_{p}^{i 1}}=\frac{2 \cos \theta_{i 1} \sin \theta_{t 1}}{\sin \left(\theta_{i 1}+\theta_{t 1}\right) \cos \left(\theta_{i 1}-\theta_{t 1}\right)} \\
& \frac{\stackrel{v}{E}^{t 2}}{E_{s}^{t 1}}=\frac{2 \cos \theta_{i 2} \sin \theta_{t 2}}{\sin \left(\theta_{i 2}+\theta_{t 2}\right)} \\
& \frac{\stackrel{\mathrm{v}}{t 2}^{\mathrm{V}^{p}}}{\mathrm{E}_{p}^{t 1}}=\frac{2 \cos \theta_{i 2} \sin \theta_{t 2}}{\sin \left(\theta_{i 2}+\theta_{t 2}\right) \cos \left(\theta_{i 2}-\theta_{t 2}\right)}
\end{aligned}
$$

The refractions also abide by Snell principle.

$$
\frac{n_{1}}{n_{2}}=\frac{\sin \theta_{t 1}}{\sin \theta_{i 1}}=\frac{\sin \theta_{i 2}}{\sin \theta_{t 2}}
$$

Then the transmission ratio of amplitude can be derived by comparing the components according to Eq.(2-5):

$$
\begin{aligned}
k & =\left[\cos \left(\theta_{i 2}-\theta_{t 2}\right) \cos \left(\theta_{i 1}-\theta_{t 1}\right)\right]^{2} \\
& =\left(\cos \theta_{i 1} \sqrt{1-\left(\frac{n_{1} \sin \theta_{i 1}}{n_{2}}\right)^{2}}+\sin \theta_{i 1} \frac{n_{1} \sin \theta_{i 1}}{n_{2}}\right)^{2}
\end{aligned}
$$

However, when the refractive index of turbulence varies with the radius there will be:

$$
\underset{E_{s}}{\stackrel{E^{t 1}}{\forall}}=\frac{2 \cos \theta_{i 1} \sin \theta_{t 1}}{\sin \left(\theta_{i 1}+\theta_{t 1}\right)}
$$




$$
\begin{aligned}
& \frac{\stackrel{\mathrm{v}}{t 1}^{t 1}}{\mathrm{~V}_{p}^{i 1}}=\frac{2 \cos \theta_{i 1} \sin \theta_{t 1}}{\sin \left(\theta_{i 1}+\theta_{t 1}\right) \cos \left(\theta_{i 1}-\theta_{t 1}\right)} \\
& \frac{{\stackrel{\mathrm{v}}{E^{t m}}}^{\mathrm{V}}}{E_{s}^{t m}}=\frac{2 \cos \theta_{i m} \sin \theta_{t m}}{\sin \left(\theta_{i m}+\theta_{t m}\right)}(m=2,3,4 \mathrm{~L}) \\
& \frac{\stackrel{v}{E}^{t m}}{\vec{V}_{p}^{t m}}=\frac{2 \cos \theta_{i m} \sin \theta_{t m}}{\sin \left(\theta_{i m}+\theta_{t m}\right) \cos \left(\theta_{i m}-\theta_{t m}\right)}(m=2,3,4 \mathrm{~L})
\end{aligned}
$$

Therefore the transmission ratio is calculated as:

$$
\begin{aligned}
& k=\left[\cos \left(\theta_{i m}-\theta_{t m}\right) \cos \left(\theta_{i m-1}-\theta_{t m-1}\right) \mathrm{L} \cos \left(\theta_{i 1}-\theta_{t 1}\right)\right]^{2} \\
& \frac{n_{m-1}}{n_{m}}=\frac{\sin \theta_{t m-1}}{\sin \theta_{i m}}
\end{aligned}
$$

Figure. 2 shows the relationship between $\mathrm{k}$ and the incident angle $\theta_{1}$ at different turbulence scales. It can be seen that no matter how large the turbulence is, when incident angle is 0 the photon penetrates the bubble directly along the diameter so the transmission ratio reaches its climax. However, this value descends rapidly when the incident angle exceeds a certain threshold. The solid line shows the situation of turbulence with $1 \mathrm{~m}$ radius and dashed line is $5 \mathrm{~m}$ while dotted line is $10 \mathrm{~m}$. It can be concluded from the trend that the larger the turbulence is, the faster its transmission ratio decreases. And the threshold of each line are 44.8, 53.2 . and 57.5 respectively. When the incident angle exceeds the threshold, the fidelity of single photons becomes worse after continuous refraction. Figure. 3 shows the relationship between $\mathrm{k}$ and $\mathrm{r}$ when the incident angle is 45 .

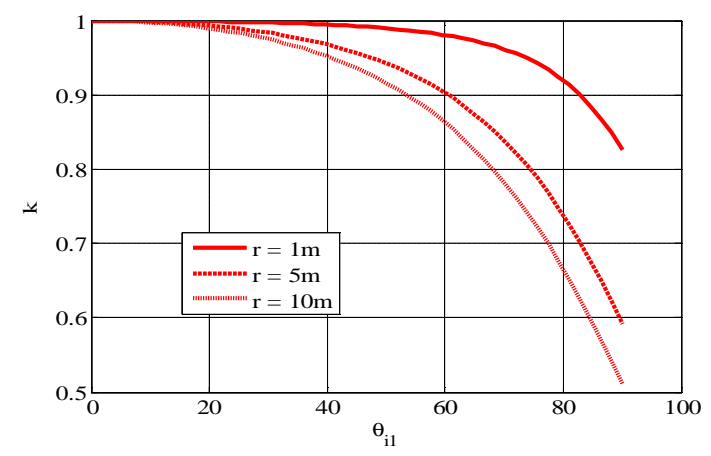

Figure 2. Relationship between transmission ratio and incident angle with different turbulence scale

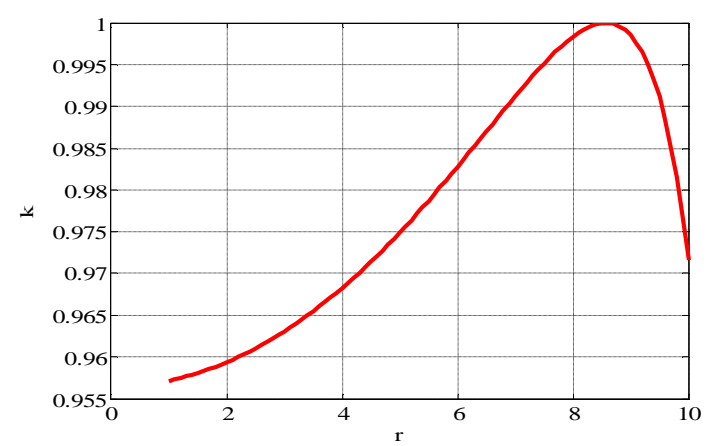

Figure 3. Relationship between transmission ratio and turbulence radius

The scale of turbulence and incident angle determine the fluctuation of transmission ratio to some extent. Here we firstly fix the incident angle at 45 . in order to figure out the optimal turbulence scale for transmitting photons. The result shows that $8 \mathrm{~m}$ is the optimal radius. Whereas, in more general situation the value of $\mathrm{k}$ will varies gradually with the change of $\mathrm{r}$ and $\theta_{i 1}$, which will induce apparent QBER. Figure.4 shows the relationship between $\mathrm{k}$ and incident angle and turbulence radius. 


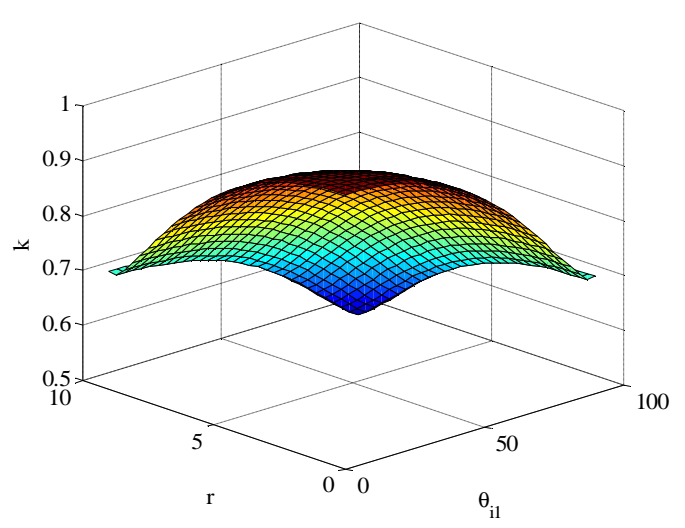

Figure 4. Relationship between $\mathrm{k}$ and incident angle and turbulence radius

In the scheme of polarization encoded BB84 protocol, Alice uses four different states to encode information on photons. The electric vector $E$ of photons at any state can be projected on $s$ direction and $p$ direction respectively. Thus the polarization state before transmission is $\alpha|P\rangle+\beta|S\rangle$. It will turn into $\alpha p|P\rangle+\beta s|S\rangle$ after refraction of turbulence. According to the analysis of Figure. 2 in general cases $s \neq p$ so the polarization state will be modified. The quantum bit error rate (QBER) due to polarization misalignment can be expressed as:

$$
Q=\frac{\left(t_{p}-t_{s}\right)^{2}}{2\left(t_{p}^{2}+t_{s}^{2}\right)}=\frac{(1-k)^{2}}{2\left(1+k^{2}\right)}
$$

Figure. 5 shows the simulation of $\mathrm{Q}$ when we take the optimal incident angle and implement the protocol at minimum turbulent influence. From this two diagrams it can be inferred that the propagating performance of single photons is unstable when $n_{2}$ changes randomly. The solid lines show the refraction of photons in turbulence with high refractive index. Although the energy will reduce greatly, the QBER increases slowly. The requirement of free space QKD can be satisfied only if the sending frequency is high enough. But the photons cannot penetrate with some probability due to total reflection and interrupt the regular key distribution process; the whole tendency coincides with the analysis of Figure.2.

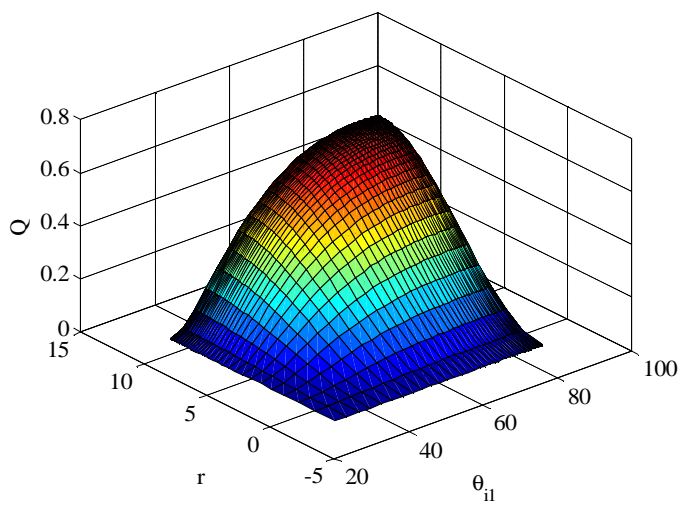

Figure 5. Relationship between $Q$ and incident angle and turbulence radius

\section{Conclusion}

In general, we focus on the performance free space QKD in turbulent environment. The propagation process of polarized photons is abstracted as continuous refraction based on turbulence bubble model. Then the relationship between incident angle and transmission ratio and polarization QBER is simulated through analyzing change of polarization state. The upper bound of QBER is derived according to relationship between key rate and transmission ratio. Furthermore, the secure range of incident angle and refractive index is figured out. The results show that the performance of free space 
QKD will be influenced by turbulence due to the refraction effect on polarization states. The requirement of QBER can be satisfied by optimizing parameters in future work.

\section{References :}

[1] Bonato,F.; Tomaello,A.; Deppo,V.D.; et al. Feasibility of satellite quantum key distribution [J] New J Phys 2009, 11, 045017.

[2] Villoresi,P.; Jennewein,T.; Tamburini,F.; et al. Experimental verification of the feasibility of a quantum channel between Space and Earth [J]. New J Phys 2008, 10, 3436-3440.

[3]. Jin,X.M.; Ren,J.G.; Yang,B.; et al. Experimental free-space quantum teleportation [J]. Nature Photon 2010, 4, 376-381.

[4]. Nauerth,S.; Moll,F.; Rau,M.; et al. Air-to-ground quantum communication [J]. Nature Photon 2013, 7, 382-386.

[5]. Min,N.; Jiaming,R.; Guang,Y.; et al. Influences of nonspherical aerosol particles and relative humidity of atmosphere on the performance of free space quantum communication [J]. Acta Phys $\operatorname{Sin} 2016,65,1-9$.

[6]. Min,N.; Yun,W.; Guang,Y.; et al. Optimal mean photon number of decoy state protocol based on chameleon self-adaptive strategy under the background of rainfall [J]. Acta Phys Sin 2016,65, 23-30.

[7]. Min,N.; Kun,G.; Guang,Y.; et al. Effect of the Atmospheric Turbulence on the Performance of Free Space Quantum Communication [J]. Acta Photonica Sinica 2016, 45, 143-149.

[8]. Dong,L.; Wang,J.X.; Li.Q.Y,; et al. Teleportation of a general two-photon state employing a polarization-entangled state with nondemolition parity analyses [C]. Quantum Inf Process 2016, 2, $1-16$.

[9]. Chong,Y.Y.; Ming,L; Peng,F.L,. Photon polarization in free-space quantum communication [J]. Journal of Beijing University of Posts and Telecommunications 2013, 36, 1-9.

[10]. Schmitt,T.; Weier,H.; Furst,M.; et al. Experimental demonstration of free-space decoy-state quantum key distribution over144 km [J]. Phys Rev Lett 2007, 98, 010504.

[11]. Lylova,A.; Kudryashov,A.; Sheldakova,J.; et al. A new method of the real-time atmospheric turbulence modeling [J]. International Conference Laser Optics 2016, R, 4-12.

[12]. Yuksel,D.; Yuksel,H.; Geometrical Monte Carlo simulation of atmospheric turbulence [C]. Proc Spie 2013, 11, 8874.

[13]. Carrasco,C.A,; Fernandez,V.; Denisenko,N.; Free-space quantum communication [M]. In Optical wireless communications; Murat,U.; Springer International Publishing: Berlin, Germany, 2016, pp. 589-607.

[14]. Charnotskii,M.; Bit error rate of focused Gaussian beams in weak oceanic turbulence [J]. JOSA A 2015, 32, 1247.

[15] Lo,H.K.; Ma,X.; Chen,K.; Decoy state quantum key distribution [J]. Phys Rev Lett 2011, 94, 230504. 